\title{
Sharing Data from Molecular Simulations
}

Mark Abraham, ${ }^{\dagger}$ Rossen Apostolov, ${ }^{\ddagger}$ Jonathan Barnoud, ${ }^{\S, \bigcirc}$ Paul Bauer, $^{\dagger}$ Christian Blau, ${ }^{\dagger}$ Alexandre M.J.J. Bonvin, ${ }^{\| \odot}$ Matthieu Chavent, ${ }^{*}, \perp \odot$ John Chodera, ${ }^{\#}$ Karmen Čndić-Jurkić, Lucie Delemotte, ${ }^{\dagger \odot}$ Helmut Grubmüller, ${ }^{\circledR}$ Rebecca J. Howard, ${ }^{\mathrm{I}, \dagger}$ E. Joseph Jordan, ${ }^{\mathrm{T}}$ Erik Lindahl, ${ }^{\mathrm{I}, \dagger}$

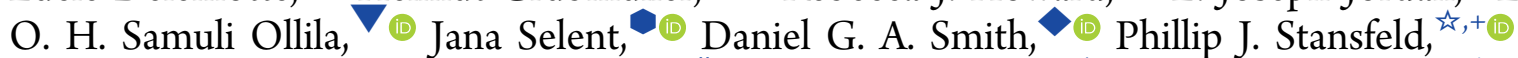
Johanna K.S. Tiemann, ${ }^{\boxplus}$ Mikael Trellet, " Christopher Woods, ${ }^{\bullet}$ and Artem Zhmurov ${ }^{\dagger}$

${ }^{\dagger}$ Science for Life Laboratory, Department of Applied Physics, KTH Royal Institute of Technology, Box 1031, SE-171 21 Solna, Sweden

${ }^{\ddagger}$ PDC Center for High Performance Computing, School of Electrical Engineering and Computer Science, KTH Royal Institute of Technology, 11428 Stockholm, Sweden

${ }^{\S}$ University of Groningen, 9712 CP Groningen, Netherlands

"Faculty of Science, Utrecht University, Bijvoet Center, Utrecht, The Netherlands

${ }^{\perp}$ IPBS, Université Paul Sabatier, F-31062 Toulouse, France

${ }^{\#}$ Computational and Systems Biology Program, Sloan Kettering Institute, Memorial Sloan Kettering Cancer Center, New York, New York 10065, United States

Open Force Field Consortium, https://openforcefield.org/consortium/

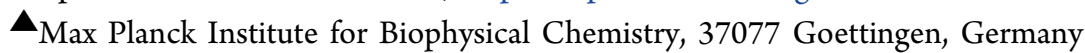

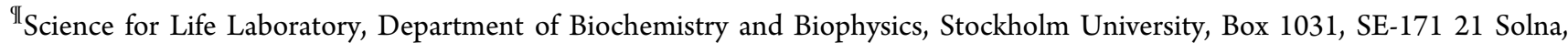
Sweden

Institute of Biotechnology, University of Helsinki, 00100 Helsinki, Finland

- Research Programme on Biomedical Informatics, Hospital del Mar Medical Research Institute (IMIM) \& Department of Experimental and Health Sciences, Pompeu Fabra University, 08002 Barcelona, Spain

The Molecular Sciences Software Institute, Blacksburg, Virginia 24060, United States

${ }^{25}$ Department of Biochemistry, University of Oxford, Oxford OX1 2JD, U.K.

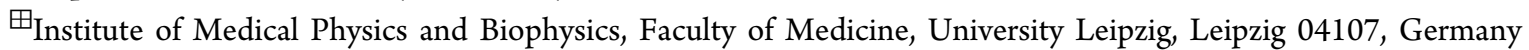

-University of Bristol, Bristol BS8 1TH, U.K.

ABSTRACT: Given the need for modern researchers to produce open, reproducible scientific output, the lack of standards and best practices for sharing data and workflows used to produce and analyze molecular dynamics (MD) simulations has become an important issue in the field. There are now multiple well-established packages to perform molecular dynamics simulations, often highly tuned for exploiting specific classes of hardware, each with strong communities surrounding them, but with very limited interoperability/transferability options. Thus, the choice of the software package often dictates the workflow for both simulation production and analysis. The level of detail in documenting the workflows and analysis code varies greatly in published work, hindering reproducibility of the reported results and the ability for other researchers to build on these studies. An increasing number of researchers are motivated to make their

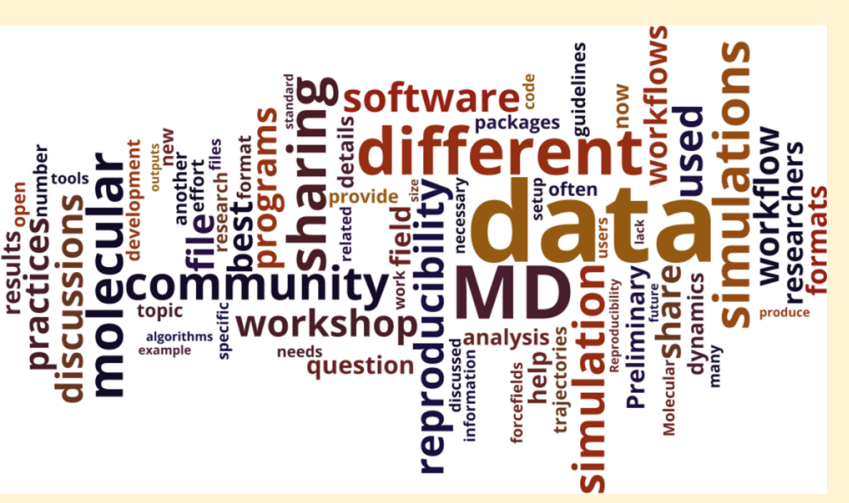
data available, but many challenges remain in order to effectively share and reuse simulation data. To discuss these and other issues related to best practices in the field in general, we organized a workshop in November 2018 (https://bioexcel.eu/events/workshop-on-sharing-data-from-molecular-simulations/). Here, we present a brief overview of this workshop and topics discussed. We hope this effort will spark further conversation in the MD community to pave the way toward more open, interoperable, and reproducible outputs coming from research studies using MD simulations.

\section{INTRODUCTION}

Molecular simulations have become increasingly powerful and accessible in recent years, due in part to the rise of $\mathrm{HPC}^{1-3}$ and
Received: August 8, 2019

Published: September 17, 2019 
GPU-powered clusters and powerful desktop computers ${ }^{4}$ as well as the development of user-friendly software to setup simulations. ${ }^{5,6}$ The underlying physical models and methods have also improved over the years to address ever more complex biological and chemical questions. ${ }^{7,8}$ Finally, the number of users and available tools is continuously increasing, as is the amount and complexity of workflows and produced outputs. ${ }^{9,10}$ In this context, defining best practices related to documentation of protocols and code used to generate and/or analyze Molecular Dynamics (MD) simulations is becoming more important than ever. ${ }^{11} \mathrm{~A}$ set of guidelines for reporting results obtained using molecular dynamics techniques and an opportunity to share data, similar to what structural biologists have achieved with the worldwide Protein Data Bank ${ }^{12}$ (wwPDB), should generally help to improve the quality, reproducibility, statistics, and reuse of the published results.

Here, we would like to focus on the term reproducibility. The definition of reproducibility and its distinction from replicability can vary between disciplines, ${ }^{13-15}$ but in this context, we will broadly define reproducibility as the ability to reimplement the workflows of published work and obtain similar behavior for observables of interest as well as define the appropriate way to measure/calculate and report these observables. ${ }^{16}$ Reproducibility is a long-standing issue for molecular modeling ${ }^{17}$ and a key step toward better reproducibility and improved collaboration is making data more accessible and workflows interoperable. This can help reduce the entry barrier for the newcomers, but it could also help the existing practitioners to focus on answering scientific questions rather than wasting time in redeveloping existing sets of parameters or translating files formats to pass from one software to another. To reach this goal, it is now necessary to overcome several difficulties:

- First, there is now a multitude of package-specific file formats and object models. This variety, although increasing the efficiency for each package, introduces limitations in the interoperability and creates friction for users juggling with various software to generate and analyze their data.

- Second, there is still a lack of exhaustive documentation related to new software development. The proliferation of various libraries and toolkits definitely opens up new avenues of research, but documenting the entire workflow from building a molecular model and parametrization to data analysis and visualization has become more complex. The method sections in publications often lack sufficient details to successfully reimplement the protocol or repeat the study from scratch, and default parameters to run a simulation may vary from one software version to another.

- Last but not least, there is no consensus to share data. The recent years have seen developments of different open data platforms, but the (ever-increasing) size of the generated trajectories makes it difficult to share simulation data efficiently. The absence of appropriate infrastructure, guidelines, and incentives further complicate the situation. ${ }^{18,19}$

In general, we are witnessing a growing effort to make science more open by researchers themselves and increasingly so by funders and journals. ${ }^{20,21}$ Soon, it may be mandatory to share data and deposit models obtained from hybrid/integrative approaches combining molecular modeling and experimental results. $^{22}$ Finding a way to consistently share data, workflows, and protocols will be thus necessary to ensure an efficient information exchange. Defining best practices and coming up with solutions should be a community effort to achieve the best outcome for everyone involved. In an effort to start a discussion around these questions, we organized a BioExcel workshop on Sharing Data from Molecular Simulations (SDMS) in Stockholm, November 2018. In this paper, we present a summary of discussions broadly focused on four topics:

- Standardization of file formats

- Streamlining molecular simulation data

- Tools for trajectory file sharing

- Reproducibility of molecular simulations

Each topic was introduced by two researchers and then openly discussed by all participants. All the presentations and the discussions were recorded and are accessible here: https:// bioexcel.eu/sdms18-recordings/. The slides for the majority of the talks can be found here: 10.5281 /zenodo.2652703.

\section{STANDARDIZATION OF FILE FORMATS}

While in structural biology the established PDB file format was stable for decades, ${ }^{12}$ the MD simulations field has a tendency to produce a multitude of input/output formats each related to one MD package. ${ }^{1,23-27}$ With the rapid growth in complexity, size, and number of macromolecular structures led by advances in experimental techniques, even the canonical PDB format is now evolving to allow rendering and analyzing larger files with a gain in performance. ${ }^{28}$ This evolution may also encourage the MD community to update its file formats to deal with larger and more heterogeneous data.

A new jointly developed format would need to be modular and flexible enough to not only take into account current but also anticipate future needs. Here arises a first question: What are the current and future needs of the MD community for such a format? While particle coordinates are the current main feature both for input and output standards, other features need to be discussed such as physical/chemical descriptions of the model, experimental data used to create the model, technical details related to the simulation (such as algorithms used, sampling method, and force field). Different formats may be used as templates such as MMTF, ${ }^{28}$ MMCIF, $^{29}$ JSON (http://www. json.org/), and TNF. ${ }^{30}$ At this workshop, we all agreed that it would be a great advance if this new standard can follow the FAIR principle: ${ }^{31}$ Findable, Accessible, Interoperable, and Reproducible/Reusable. Many details remain to be discussed, and the standardization question cannot be solved in one workshop with only a small sample of the MD community; it needs to be discussed by all the main software developers joined with users to ensure usability. To do so, another workshop will be held soon in New York to further discuss the question of file format and MD package interoperability: https://molssi.org/ 2019/07/29/molssi-workshop-molecular-dynamics-softwareinteroperability/.

For further details and discussions, interested readers can watch associated videos from the 2018 workshop:

- Introduction of the topic by Mark Abraham (https:// youtu.be/2S3qjBIE6Y4)

- Preliminary talk I by Erik Lindahl (https://youtu.be/ Hvy8-gyTmj8)

- Preliminary talk II from Alexandre Bonvin (https:// youtu.be/48Eb2MLHoYU)

- Breakout discussions presented by Phillip Stansfeld, Mikael Trellet, Daniel Smith, and Johanna Tiemann (https://youtu.be/4fnV5EFXDpc) 


\section{STREAMLINING MOLECULAR SIMULATIONS DATA}

The MD simulation is often not a means and an end in itself but instead is run as part of a larger workflow. Such workflows involve joining together the output of many independent programs, such as those used for parametrizing molecules, those for performing molecular dynamics, and those for trajectory analysis. Managing the data movement between different programs in this workflow is challenging for several reasons:

1. The file formats used by different programs in the workflow may be incompatible, thereby preventing certain combinations of tools from being used together.

2. The features and force fields supported by different programs in the workflow may be incompatible, thereby forcing researchers to choose algorithms and force fields based on software compatibility rather than for good scientific reasons.

3. Different programs may implement features or force fields in different ways, thereby meaning that the results of running the workflow will depend on the exact combination of programs (and possibly program versions) used. It is generally not possible to mix-andmatch different programs and get the same results.

These challenges have forced researchers to develop workflows using specific software packages and specific force fields. This creates divisions within the community and makes it difficult to write workflows that function equally well across a number of force fields and a number of different software packages.

One of the solutions to this problem is the development of programs that convert/handle molecular information between the different file formats such as VMD, ${ }^{32}$ cpptraj, ${ }^{33}$ MDAnalysis, ${ }^{34,35}$ mdtraj, ${ }^{36}$ LOOS, ${ }^{37,38}$ and many others for trajectory analysis and TopoGromacs, ${ }^{39}$ CHARMM-GUI, ${ }^{40}$ CHAMBER, ${ }^{41}$ ParmEd (http://parmed.github.io/ParmEd/html/ index.html\#), InterMol ${ }^{42}$ (https://github.com/shirtsgroup/ InterMol), and others for topology generation and editing. The aim of these programs is to translate as much information as possible from one molecular file format into another. One recent example is BioSimSpace (https://biosimspace.org/), which provides wrappers that simplify the generation of the command files that are used to control the running of simulations. This allows researchers to write workflows that are independent of the choice of the underlying packages used to perform the simulation. BioSimSpace aims to run all stages of the workflow using the simulation software installed on the researcher's computer that is compatible with the force field chosen for the specific calculation.

While translators and program wrappers like ParmEd and BioSimSpace solve some of these problems, they are not a universal solution. They do not solve the issue that different simulation programs use different algorithms (or interpretations of algorithms, for example, different implementations of thermostats or integrators) or that different programs store and represent molecular information in different ways (e.g., SHAKE information for constraining bonds is represented in the molecular topology in GROMACS, while it is a simulation command parameter in NAMD and AMBER). This means MD properties/observables computed with one package will be systematically different by an often small but statistically significant amount from those computed with a different package as shown for free energy calculations. ${ }^{43}$ Thus, the version and name of the $\mathrm{MD}$ program used to produce a simulation result will affect that result and must be reported accordingly. Furthermore, MD simulation outputs are mainly trajectories which (1) represent ensemble averages and (2) are chaotic in that small differences in initial conditions cause large differences in the subsequent dynamics ("butterfly effect"). This adds another layer of complexity and needs also a consensus on how to further analyze/process these trajectories to provide the final quantities of interest.

The recordings of this session can be found here:

- Introduction to the topic by John Chodera (https:// youtu.be/6xOfN0y_uoQ)

- Preliminary talk I by Philip Stansfeld (https://youtu.be/ YPYeujSD-6Y)

- Preliminary talk II by Christopher Woods (https://youtu. be/w1d1xtbGhHc)

- Breakout discussions presented by Christian Blau, Christopher Woods, Jonathan Barnoud, and Mark Abraham (https://youtu.be/Z-JfBU3Emug)

\section{TOOLS FOR TRAJECTORY FILE SHARING}

The benefits of sharing data together with the peer-reviewed publication, preprint, or as a self-standing research output seem to be many-from receiving additional credit for one's work to improving reproducibility, reusability, or offering potentially new avenues of research. ${ }^{20,44}$ Some disciplines, such as protein crystallography or genomics, have open data practices well integrated into their workflow, with metadata being collected throughout the workflow, and those practices are a de facto standard in scholarly communication. However, data sharing in the MD community still has not become widely adopted because best practice guidelines or journal recommendations on how to share MD simulations are yet to be established and adopted by the whole community. Making data sharing a standard practice in the field faces both technical and cultural challenges, although these are currently being tackled by some ongoing initiatives and solutions. $^{20,45,46}$ Thus, the development of best practices and guidelines for simulation data sharing will be of tremendous value, especially if created with the FAIR principles in mind. ${ }^{31}$ To do so, we need to address several important questions regarding what data should be shared, how and where.

Answering to the what data question would need longer discussions not limited to a small group of individuals but involving the whole community and especially all the $\mathrm{MD}$ packages (another workshop will be held soon to help starting to answer to this question: https://molssi.org/2019/07/29/ mols si-workshop-molecular-dynamics-softwareinteroperability/). The emergence of dedicated tools is now helping to answer to the how question. Software such as MDsrv, ${ }^{47} \mathrm{HTMoL}^{48}{ }^{48} \mathrm{Mol}^{*}$ (https://molstar.org), and Molmil $^{49}$ are now taking advantage of the WebGL API for sharing trajectories through interactive visualization on the web. ${ }^{50}$

Other fields of research can help us to answer to the where question. Existing databanks, such as $w_{w P D B}{ }^{51}$ and Galaxy (https://usegalaxy.org), have been recognized by the scientific community. However, the establishment of an analogous, specialized platform for MD data, poses a great challenge, given the current lack of long-term support for the infrastructure projects of this kind. It is not clear yet who should be responsible for building such platform and how this infrastructure could be funded in a sustainable way, preferably without relying on shortterm research grants, to cover the costs of development, maintenance, and data hosting. In the meantime, community- 
driven, special-purpose platforms like the GPCRmd (http:// www.gpcrmd.org), Lipidbook ${ }^{52}$ and NMRlipids ${ }^{45}$ (http:// nmrlipids.blogspot.com), Ligandbook, ${ }^{53}$ MoDEL, ${ }^{54}$ and BIGNASim $^{55}$ lead the way, providing specialized platforms for deposition and analysis of $G$ protein-coupled receptors (GPCR), lipids, small molecules, proteins, and nucleic acids, respectively. General data sharing resources like Zenodo (https://zenodo.org), FigShare (https://figshare.com), Open Science Framework (https://osf.io), and others also provide an opportunity for every researcher to deposit their simulation files and trajectories. Nevertheless, those resources may, sometimes, not provide enough space to sustainably store MD simulations outputs (with file size limits ranging between 5 and 50 GB).

To establish an efficient sharing culture, a systematic approach to developing tools and sharing guidelines is necessary, with the participation of the entire community in such activities and efforts. An open and inclusive discussion about best practices in data sharing and identification of short-term solutions based on the currently available frameworks and tools, as well as developing a strategy and requirements for future solutions bespoke to the MD community and their needs is necessary. More details about the discussions taking place at the workshop can be found in the following videos:

- Introduction to the topic by Daniel Smith (https://youtu. be/mvesL9Y_9xU)

- Preliminary talk I by Johanna Tiemann (https://youtu. be/VOT6fEc7Iuc)

- Preliminary talk II by Jana Selent (https://youtu.be/ TVS75j48mQ8)

- Breakout discussions presented by John Chodera, Karmen Čondić-Jurkić, Samuli Olllila, and Lucie Delemotte (https://youtu.be/UIslisntUPY)

\section{REPRODUCIBILITY OF MOLECULAR SIMULATIONS}

MD simulations are chaotic, and as such, the definition of reproducible results is nontrivial. First, the distinction between repeatability (by the same team and the same computational setup), replicability (by a different team and the same computational setup), and reproducibility (by a different team and with a different experimental setup) should be made. ${ }^{14}$ Differences in outputs from these three perspectives may indicate different types of errors (bugs in software, human errors, or different choices along the workflow-choice of code, force field, system setup, and more). The variability of parameters and dependence of the final results on both software and hardware makes it complicated (but also often unnecessary) to achieve the exact replication/repetition of any given setup, and untangling all the effects would be a difficult task. Focusing on a set of observables that can be calculated and preferably validated against experiments might be a better way of approaching reproducibility in this particular field. Similarly, focusing at observables which, despite the underlying chaoticity of the detailed dynamics, are reproducible without too large variation might be beneficial. Reaching an agreement on which observables we should aim to reproduce and how to properly calculate and report these values is thus desirable. For this, educational efforts are needed: best practice dissemination in terms of calculating statistical properties, for example, are crucial. ${ }^{16}$ Coming up with standard benchmarks would also help, where the performance of different software/force field combinations for selected tasks could be compared.
In practice, data sharing would help with replicability and reproducibility. Practical challenges come from the size of data sets. However, one can envision sharing at least minimal data sets to improve the following:

- Methods reproducibility. Provide sufficient details to replicate the study; this is in principle already done in publications, but authors, reviewers, and editors should pay special attention to the question, and direct sharing of all input files should be mandatory,

- Raw data reproducibility. Share a minimum amount of data in the form of MD simulation snapshots or, even better, whole trajectories on existing data sharing repositories-Zenodo, Figshare, OSF.

- Results and inferential reproducibility: Share among other analysis code, pipeline/workflow and example used.

Inspiration can be found in other research fields (e.g., genomics ${ }^{56}$ or proteomics ${ }^{57}$ ), and existing dedicated initiatives, like MemProtMD ${ }^{58}$ (http://memprotmd.bioch.ox.ac.uk), the NMRlipids project (www.nmrlipids.blogspot.fi), and GPCRmd (http://www.gpcrmd.org), show that small groups of people focused on a specific topic can create the necessary structure to share even large data sets in an efficient way. For further details and discussions interested readers can watch associated videos:

- Introduction to the topic by Karmen Čondić-Jurkić (https://youtu.be/lUTQgOXDEP8)

- Preliminary talk I by Helmut Grubmüller (https:/ / youtu. be/cliVmGlrKag)

- Preliminary talk II by Samuli Ollila (https://youtu.be/ 46s33SonsiU)

- Breakout discussions presented by Mikael Trellet, Alexandre Bonvin, Mark Abraham, and Christopher Woods (https://youtu.be/ex0_bqmJwE8)

This article summarizes the discussions started during the workshop held in Stockholm in November 2018. As may be noted by the reader, these discussions have not solved the issues about sharing data that our field is facing. Of course, this has never been the goal of such a small workshop. This workshop was intended to start asking relevant questions. Thus, this document (and the videos associated) can be seen as a roadmap for future developments. It is now crucial to build a community responsible for transforming these ideas into actions. This community needs to represent a diversity of perspectives by including both $\mathrm{MD}$ users and developers, newcomers, and more seasoned practitioners, $\mathrm{PhD}$ students and postdocs, who are performing MD simulations on a daily basis, and PIs, who may hold the bigger picture views. As a community building effort, we are planning to regularly organize more specific workshops aiming to address some of the issues raised in this article or to expand the scope of newly recognized problems. Of course, the structure of the workshops limits the number of participants but care will be taken to ensure the aforementioned diversity of perspectives and roles in the field. In an effort to include as many users as possible in this discussion, the best practices guidelines that will emerge from these workshops will be submitted to the Living Journal of Computational Molecular Science (http:// www.livecomsjournal.org/). This journal “...provides a venue where authors can submit living documents that are updated on an ongoing basis as websites or Wikipedia articles could be, but which still have clear authorship and provide a mechanism for authors to get publication credit for their work." ${ }^{59}$ Hence, researchers interested to help us shape new practices to share data will be able to provide their feedback or directly contribute 
to the forthcoming document (as per the general idea laid out here: https://livecomsjournal.github.io/about/paper_code/). We hope that our work will act as a first step in a communitydriven process of defining best practices for tool development and application in the molecular dynamics field.

\section{AUTHOR INFORMATION}

\section{Corresponding Author}

*Email: matthieu.chavent@ipbs.fr. Twitter: @Matth_Chavent. ORCID

Alexandre M.J.J. Bonvin: 0000-0001-7369-1322

Matthieu Chavent: 0000-0003-4524-4773

John Chodera: 0000-0003-0542-119X

Lucie Delemotte: 0000-0002-0828-3899

Helmut Grubmüller: 0000-0002-3270-3144

O. H. Samuli Ollila: 0000-0002-8728-1006

Jana Selent: 0000-0002-1844-4449

Daniel G. A. Smith: 0000-0001-8626-0900

Phillip J. Stansfeld: 0000-0001-8800-7669

Artem Zhmurov: 0000-0002-4414-8352

\section{Present Addresses}

OIntangible Realities Laboratory, University of Bristol, UK.

${ }^{+}$School of Life Sciences \& Department of Chemistry, The University of Warwick, Coventry, CV4 7AL.

\section{Funding}

The workshop was supported by BioExcel Centre of Excellence (www.bioexcel.eu).

\section{Notes}

The authors declare no competing financial interest.

Link to the SDMS18 recordings: https://bioexcel.eu/sdms18recordings/. Discussions from Twitter can be retrieved/ extended by using the hashtag \#SDMS18. Several participants from this workshop can be contacted/followed on Twitter: @ the_mabraham,@jbarnoud, @amjjbonvin, @Matth_Chavent, @jchodera, @karmecon, @DelemotteLab, @CompBioPhys, @ eriklindahl, @NMRlipids, @dga_smith, @pstansfeld, @j0kaso, @chryswoods.

\section{ACKNOWLEDGMENTS}

This work was supported by BioExcel Centre of Excellence (www.bioexcel.eu) funded by the European Union contracts H2020-INFRAEDI-02-2018-823830 and H2020-EINFRA2015-1-675728. M.C. acknowledges support from CNRSMITI grants PEPS MPI 2018 and "Modélisation du vivant" 2019. This work was supported by grants from the Gustafsson Foundation and Science for Life Laboratory to L.D. H.G. has been supported by Max Planck Society and the German Research Foundation (DFG), Cluster of excellence Multiscale Imaging, and the DFG priority programmes 1648, 755, and 803. O.H.S.O. acknowledges financial support from Academy of Finland (315596). D.G.A.S. thanks the National Science Foundation for support under Grant No. ACI-1547580. P.J.S. would like to thank Wellcome $[208361 / \mathrm{Z} / 17 / \mathrm{Z}]$, the BBSRC $[\mathrm{BB} / \mathrm{P} 01948 \mathrm{X} / 1, \mathrm{BB} / \mathrm{R} 002517 / 1, \mathrm{BB} / \mathrm{S} 003339 / 1$, and BB/ I019855/1] and MRC [MR/S009213/1] for funding. J.K.S.T. acknowledges support from the Deutsche Forschungsgemeinschaft (DFG) HI1502/1-2. C.W. acknowledges support from the EPSRC (EP/N018591/1). We thank Oliver Beckstein and David Mobley for their careful reading and their comments.

\section{REFERENCES}

(1) Abraham, M. J.; Murtola, T.; Schulz, R.; Páll, S.; Smith, J. C.; Hess, B.; Lindahl, E. GROMACS: High Performance Molecular Simulations Through Multi-Level Parallelism From Laptops to Supercomputers. SoftwareX 2015, 1-2, 19-25.

(2) Lagardère, L.; Jolly, L.-H.; Lipparini, F.; Aviat, F.; Stamm, B.; Jing, Z. F.; Harger, M.; Torabifard, H.; Cisneros, G. A.; Schnieders, M. J.; Gresh, N.; Maday, Y.; Ren, P. Y.; Ponder, J. W.; Piquemal, J.-P. TinkerHP: a Massively Parallel Molecular Dynamics Package for Multiscale Simulations of Large Complex Systems with Advanced Point Dipole Polarizable Force Fields. Chem. Sci. 2018, 9, 956-972.

(3) Jung, J.; Nishima, W.; Daniels, M.; Bascom, G.; Kobayashi, C.; Adedoyin, A.; Wall, M.; Lappala, A.; Phillips, D.; Fischer, W.; Tung, C. S.; Schlick, T.; Sugita, Y.; Sanbonmatsu, K. Y. Scaling Molecular Dynamics Beyond 100,000 Processor Cores for Large-Scale Biophysical Simulations. J. Comput. Chem. 2019, 40, 1919.

(4) Stone, J. E.; Hardy, D. J.; Ufimtsev, I. S.; Schulten, K. GPUAccelerated Molecular Modeling Coming of Age. J. Mol. Graphics Modell. 2010, 29, 116-125.

(5) Doerr, S.; Harvey, M. J.; Noé, F.; De Fabritiis, G. HTMD: HighThroughput Molecular Dynamics for Molecular Discovery. J. Chem. Theory Comput. 2016, 12, 1845-1852.

(6) Jo, S.; Cheng, X.; Lee, J.; Kim, S.; Park, S. J.; Patel, D. S.; Beaven, A. H.; Lee, K. I.; Rui, H.; Park, S.; Lee, H. S.; Roux, B.; MacKerell, A. D.; Klauda, J. B.; Qi, Y.; Im, W. CHARMM-GUI 10 Years for Biomolecular Modeling and Simulation. J. Comput. Chem. 2017, 38, 1114-1124.

(7) Marrink, S. J.; Corradi, V.; Souza, P. C. T.; Ingólfsson, H. I.; Tieleman, D. P.; Sansom, M. S. P. Computational Modeling of Realistic Cell Membranes. Chem. Rev. 2019, 119, 6184.

(8) Bottaro, S.; Lindorff-Larsen, K. Biophysical Experiments and Biomolecular Simulations: a Perfect Match? Science 2018, 361, 355360.

(9) Im, W.; Liang, J.; Olson, A.; Zhou, H.-X.; Vajda, S.; Vakser, I. A. Challenges in Structural Approaches to Cell Modeling. J. Mol. Biol. 2016, 428, 2943-2964.

(10) Chavent, M.; Duncan, A. L.; Sansom, M. S. Molecular Dynamics Simulations of Membrane Proteins and Their Interactions: From Nanoscale to Mesoscale. Curr. Opin. Struct. Biol. 2016, 40, 8-16.

(11) Elofsson, A.; Hess, B.; Lindahl, E.; Onufriev, A.; Van Der Spoel, D.; Wallqvist, A. Ten Simple Rules on How to Create Open Access and Reproducible Molecular Simulations of Biological Systems. PLoS Comput. Biol. 2019, 15, e1006649.

(12) Berman, H. M.; Kleywegt, G. J.; Nakamura, H.; Markley, J. L. The Protein Data Bank at 40: Reflecting on the Past to Prepare for the Future. Structure (Oxford, U. K.) 2012, 20, 391-396.

(13) Plesser, H. E. Reproducibility vs. Replicability: a Brief History of a Confused Terminology. Front Neuroinform 2018, 11, 76.

(14) Hinsen, K. A Platform for Publishing and Archiving ComputerAided Research. F1000Research 2014, 3, 289.

(15) Barba, L. A. Terminologies for Reproducible Research. arXiv.org 2018, 1802.03311.

(16) Grossfield, A.; Patrone, P. N.; Roe, D. R.; Schultz, A. J.; Siderius, D. W.; Zuckerman, D. M. Best Practices for Quantification of Uncertainty and Sampling Quality in Molecular Simulations [Article v1.0]. LiveCoMS 2019, DOI: 10.33011/livecoms.1.1.5067.

(17) Walters, W. P. Modeling, Informatics, and the Quest for Reproducibility. J. Chem. Inf. Model. 2013, 53, 1529-1530.

(18) Graham, S. C.; Nagar, B.; Privé, G. G.; Deane, J. E. Molecular Models Should Not Be Published Without the Corresponding Atomic Coordinates. Proc. Natl. Acad. Sci. U. S. A. 2019, 116, 11099-11100.

(19) Romero, R.; Yuen, T.; New, M. I.; Zaidi, M.; Haider, S. Reply to Graham Et Al.: in Silico Atomistic Coordinates and Molecular Dynamics Simulation Trajectories of the Glucocerebrosidase-Saposin C Complex. Proc. Natl. Acad. Sci. U. S. A. 2019, 116, 11101-11102.

(20) Data Sharing and the Future of Science. Nat. Commun. 2018, 9, 2817.

(21) Bentley, N.; Aufreiter, M.; Maciocci, G. Introducing eLife's First Computationally Reproducible Article. eLIFE 2019. 
(22) Burley, S. K.; Kurisu, G.; Markley, J. L.; Nakamura, H.; Velankar, S.; Berman, H. M.; Sali, A.; Schwede, T.; Trewhella, J. PDB-Dev: a Prototype System for Depositing Integrative/Hybrid Structural Models. Structure (Oxford, U. K.) 2017, 25, 1317-1318.

(23) Phillips, J. C.; Braun, R.; Wang, W.; Gumbart, J.; Tajkhorshid, E.; Villa, E.; Chipot, C.; Skeel, R. D.; Kalé, L.; Schulten, K. Scalable Molecular Dynamics with NAMD. J. Comput. Chem. 2005, 26, 17811802.

(24) Brooks, B. R.; Brooks, C. L.; Mackerell, A. D.; Nilsson, L.; Petrella, R. J.; Roux, B.; Won, Y.; Archontis, G.; Bartels, C.; Boresch, S.; Caflisch, A.; Caves, L.; Cui, Q.; Dinner, A. R.; Feig, M.; Fischer, S.; Gao, J.; Hodoscek, M.; Im, W.; Kuczera, K.; Lazaridis, T.; Ma, J.; Ovchinnikov, V.; Paci, E.; Pastor, R. W.; Post, C. B.; Pu, J. Z.; Schaefer, M.; Tidor, B.; Venable, R. M.; Woodcock, H. L.; Wu, X.; Yang, W.; York, D. M.; Karplus, M. CHARMM: the Biomolecular Simulation Program. J. Comput. Chem. 2009, 30, 1545-1614.

(25) Salomon Ferrer, R.; Case, D. A.; Walker, R. C. An Overview of the Amber Biomolecular Simulation Package. Wiley Interdisciplinary Reviews: Computational Molecular Science 2013, 3, 198-210.

(26) Rackers, J. A.; Wang, Z.; Lu, C.; Laury, M. L.; Lagardère, L.; Schnieders, M. J.; Piquemal, J.-P.; Ren, P.; Ponder, J. W. Tinker 8: Software Tools for Molecular Design. J. Chem. Theory Comput. 2018, 14, 5273-5289.

(27) Eastman, P.; Friedrichs, M. S.; Chodera, J. D.; Radmer, R. J.; Bruns, C. M.; Ku, J. P.; Beauchamp, K. A.; Lane, T. J.; Wang, L.-P.; Shukla, D.; Tye, T.; Houston, M.; Stich, T.; Klein, C.; Shirts, M. R.; Pande, V. S. OpenMM 4: a Reusable, Extensible, Hardware Independent Library for High Performance Molecular Simulation. J. Chem. Theory Comput. 2013, 9, 461-469.

(28) Bradley, A. R.; Rose, A. S.; Pavelka, A.; Valasatava, Y.; Duarte, J. M.; Prlić, A.; Rose, P. W. MMTF-an Efficient File Format for the Transmission, Visualization, and Analysis of Macromolecular Structures. PLoS Comput. Biol. 2017, 13, e1005575.

(29) Bourne, P. E.; Berman, H. M.; McMahon, B.; Watenpaugh, K. D.; Westbrook, J. D.; Fitzgerald, P. Macromolecular Crystallographic Information File. Methods Enzymol. 1997, 277, 571-590.

(30) Lundborg, M.; Apostolov, R.; Spangberg, D.; Gardenas, A.; Van Der Spoel, D.; Lindahl, E. An Efficient and Extensible Format, Library, and API for Binary Trajectory Data From Molecular Simulations. J. Comput. Chem. 2014, 35, 260-269.

(31) Wilkinson, M. D.; Dumontier, M.; Aalbersberg, I. J. J.; Appleton, G.; Axton, M.; Baak, A.; Blomberg, N.; Boiten, J.-W.; da Silva Santos, L. B.; Bourne, P. E.; Bouwman, J.; Brookes, A. J.; Clark, T.; Crosas, M.; Dillo, I.; Dumon, O.; Edmunds, S.; Evelo, C. T.; Finkers, R.; GonzalezBeltran, A.; Gray, A. J. G.; Groth, P.; Goble, C.; Grethe, J. S.; Heringa, J.; 't Hoen, P. A. C.; Hooft, R.; Kuhn, T.; Kok, R.; Kok, J.; Lusher, S. J.; Martone, M. E.; Mons, A.; Packer, A. L.; Persson, B.; Rocca-Serra, P.; Roos, M.; van Schaik, R.; Sansone, S.-A.; Schultes, E.; Sengstag, T.; Slater, T.; Strawn, G.; Swertz, M. A.; Thompson, M.; van der Lei, J.; van Mulligen, E.; Velterop, J.; Waagmeester, A.; Wittenburg, P.; Wolstencroft, K.; Zhao, J.; Mons, B. The FAIR Guiding Principles for Scientific Data Management and Stewardship. Sci. Data 2016, 3, 160018 .

(32) Humphrey, W.; Dalke, A.; Schulten, K. VMD: Visual Molecular Dynamics. J. Mol. Graphics 1996, 14, 33.

(33) Roe, D. R.; Cheatham, T. E. PTRAJ and CPPTRAJ: Software for Processing and Analysis of Molecular Dynamics Trajectory Data. J. Chem. Theory Comput. 2013, 9, 3084-3095.

(34) Michaud-Agrawal, N.; Denning, E. J.; Woolf, T. B.; Beckstein, O. MDAnalysis: a Toolkit for the Analysis of Molecular Dynamics Simulations. J. Comput. Chem. 2011, 32, 2319-2327.

(35) Gowers, R.; Linke, M.; Barnoud, J.; Reddy, T.; Melo, M.; Seyler, S.; Domański, J.; Dotson, D.; Buchoux, S.; Kenney, I.; Beckstein, O. MDAnalysis: a Python Package for the Rapid Analysis of Molecular Dynamics Simulations. SciPy 2016, 98-105.

(36) McGibbon, R. T.; Beauchamp, K. A.; Harrigan, M. P.; Klein, C.; Swails, J. M.; Hernández, C. X.; Schwantes, C. R.; Wang, L.-P.; Lane, T. J.; Pande, V. S. MDTraj: a Modern Open Library for the Analysis of Molecular Dynamics Trajectories. Biophys. J. 2015, 109, 1528-1532.
(37) Romo, T. D.; Grossfield, A. LOOS: an Extensible Platform for the Structural Analysis of Simulations. Conf Proc. IEEE Eng. Med. Biol. Soc. 2009, 2009, 2332-2335.

(38) Romo, T. D.; Leioatts, N.; Grossfield, A. Lightweight Object Oriented Structure Analysis: Tools for Building Tools to Analyze Molecular Dynamics Simulations. J. Comput. Chem. 2014, 35, 23052318.

(39) Vermaas, J. V.; Hardy, D. J.; Stone, J. E.; Tajkhorshid, E.; Kohlmeyer, A. TopoGromacs: Automated Topology Conversion From CHARMM to GROMACS Within VMD. J. Chem. Inf. Model. 2016, 56, $1112-1116$.

(40) Lee, J.; Cheng, X.; Swails, J. M.; Yeom, M. S.; Eastman, P. K.; Lemkul, J. A.; Wei, S.; Buckner, J.; Jeong, J. C.; Qi, Y.; Jo, S.; Pande, V. S.; Case, D. A.; Brooks, C. L., III; MacKerell, A. D., Jr.; Klauda, J. B.; Im, W. CHARMM-GUI Input Generator for NAMD, GROMACS, AMBER, OpenMM, and CHARMM/OpenMM Simulations Using the CHARMM36 Additive Force Field. J. Chem. Theory Comput. 2016, $12,405-413$.

(41) Crowley, M. F.; Williamson, M. J.; Walker, R. C. CHAMBER: Comprehensive Support for CHARMM Force Fields Within the AMBER Software. Int. J. Quantum Chem. 2009, 109, 3767-3772.

(42) Shirts, M. R.; Klein, C.; Swails, J. M.; Yin, J.; Gilson, M. K.; Mobley, D. L.; Case, D. A.; Zhong, E. D. Lessons Learned From Comparing Molecular Dynamics Engines on the SAMPL5 Dataset. J. Comput.-Aided Mol. Des. 2017, 31, 147-161.

(43) Loeffler, H. H.; Bosisio, S.; Duarte Ramos Matos, G.; Suh, D.; Roux, B.; Mobley, D. L.; Michel, J. Reproducibility of Free Energy Calculations Across Different Molecular Simulation Software Packages. J. Chem. Theory Comput. 2018, 14 (11), 5567-5582.

(44) Woelfle, M.; Olliaro, P.; Todd, M. H. Open Science Is a Research Accelerator. Nat. Chem. 2011, 3, 745-748.

(45) Botan, A.; Favela-Rosales, F.; Fuchs, P. F. J.; Javanainen, M.; Kanduč, M.; Kulig, W.; Lamberg, A.; Loison, C.; Lyubartsev, A.; Miettinen, M. S.; Monticelli, L.; Määttä, J.; Ollila, O. H. S.; Retegan, M.; Róg, T.; Santuz, H.; Tynkkynen, J. Toward Atomistic Resolution Structure of Phosphatidylcholine Headgroup and Glycerol Backbone at Different Ambient Conditions. J. Phys. Chem. B 2015, 119, 1507515088.

(46) Promoting Transparency and Reproducibility in Enhanced Molecular Simulations. Nat. Methods 2019, 16, 670-673. DOI: $10.1038 / \mathrm{s} 41592-019-0506-8$.

(47) Tiemann, J. K. S.; Guixà-González, R.; Hildebrand, P. W.; Rose, A. S. MDsrv: Viewing and Sharing Molecular Dynamics Simulations on the Web. Nat. Methods 2017, 14, 1123-1124.

(48) Carrillo-Tripp, M.; Alvarez-Rivera, L.; Lara-Ramírez, O. I.; Becerra-Toledo, F. J.; Vega-Ramírez, A.; Quijas-Valades, E.; GonzálezZavala, E.; González-Vázquez, J. C.; García-Vieyra, J.; Santoyo-Rivera, N. B.; Chapa-Vergara, S. V.; Meneses-Viveros, A. HTMoL: Full-Stack Solution for Remote Access, Visualization, and Analysis of Molecular Dynamics Trajectory Data. J. Comput.-Aided Mol. Des. 2018, 32, 869876.

(49) Bekker, G.-J.; Nakamura, H.; Kinjo, A. R. Molmil: a Molecular Viewer for the PDB and Beyond. J. Cheminf. 2016, 8, 42.

(50) Hildebrand, P. W.; Rose, A. S.; Tiemann, J. K. S. Bringing Molecular Dynamics Simulation Data Into View. Trends Biochem. Sci. 2019, DOI: 10.1016/j.tibs.2019.06.004.

(51) Berman, H.; Henrick, K.; Nakamura, H. Announcing the Worldwide Protein Data Bank. Nat. Struct. Mol. Biol. 2003, 10, 980.

(52) Domański, J.; Stansfeld, P. J.; Sansom, M. S. P.; Beckstein, O. Lipidbook: a Public Repository for Force-Field Parameters Used in Membrane Simulations. J. Membr. Biol. 2010, 236, 255-258.

(53) Domański, J.; Beckstein, O.; Iorga, B. I. Ligandbook - an Online Repository for Small and Drug-Like Molecule Force Field Parameters. Bioinformatics 2017, btx037.

(54) Meyer, T.; D’Abramo, M.; Hospital, A.; Rueda, M.; Ferrer-Costa, C.; Pérez, A.; Carrillo, O.; Camps, J.; Fenollosa, C.; Repchevsky, D.; Gelpí, J. L.; Orozco, M. MoDEL (Molecular Dynamics Extended Library): a Database of Atomistic Molecular Dynamics Trajectories. Structure (Oxford, U. K.) 2010, 18, 1399-1409. 
(55) Hospital, A.; Andrio, P.; Cugnasco, C.; Codo, L.; Becerra, Y.; Dans, P. D.; Battistini, F.; Torres, J.; Goñi, R.; Orozco, M.; Gelpí, J. L. BIGNASim: a NoSQL Database Structure and Analysis Portal for Nucleic Acids Simulation Data. Nucleic Acids Res. 2016, 44, D272D278.

(56) Kaye, J.; Heeney, C.; Hawkins, N.; de Vries, J.; Boddington, P. Data Sharing in Genomics - Re-Shaping Scientific Practice. Nat. Rev. Genet. 2009, 10, 331-335.

(57) Martens, L.; Vizcaíno, J. A. A Golden Age for Working with Public Proteomics Data. Trends Biochem. Sci. 2017, 42, 333-341.

(58) Stansfeld, P. J.; Goose, J. E.; Caffrey, M.; Carpenter, E. P.; Parker, J. L.; Newstead, S.; Sansom, M. S. P. MemProtMD: Automated Insertion of Membrane Protein Structures Into Explicit Lipid Membranes. Structure (Oxford, U. K.) 2015, 23, 1350-1361.

(59) Mobley, D. L.; Shirts, M. R.; Zuckerman, D. M. Why We Need the Living Journal of Computational Molecular Science. LiveCoMS 2019, 1, 2031. 